\title{
Control System Design of Anti-rolling Tank Swing Bench Using BP Neural Network PID Based on LabVIEW
}

\author{
Lihua Liang, Mingxiao Sun, Songtao Zhang, Yu Wen, Peng Zhao, Jia Yuan \\ College of Automation, Harbin Engineering University, Harbin 150001, China \\ sunmingxiao1986@163.com
}

\begin{abstract}
The anti-rolling tank swing bench is a typical hydraulic position servo system. Its function is to simulate the ship roll motion and to verify the performance of the antirolling tank. Generally, the PID control method can achieve an ideal control effect. But the uncertainties, such as the couplings and the nonlinearty of the swing bench, make it difficult to establish the precise mathematical model of system. During experiments, the different disturbance torques are generated according to the different dimension scales of the anti-rolling tanks. Changes of the natural characteristics of the system are caused by changes of load, and the fixed parameters of PID controller are difficult to continue to ensure a good control effect. In order to reduce the influences caused by the factors mentioned above, a BP neural network PID control system based on virtual instrument LabVIEW is designed. By simulating the responses of the system in real time control, the verification tests using actual system are avoided. Finally, the experiments of swing bench are carried out. The results show that the controller can ensure the control accuracy and stability. The parameters tuning process efficiency is improved. The operation is convenient. The data acquisition process is simple and practical.
\end{abstract}

Keywords: anti-rolling tank swing bench; hydraulic servo system; BP neural network PID control; parameters tuning; LabVIEW

\section{Introduction}

When a ship sails on the sea, the roll motion is caused by waves. Rolling not only has an adverse effect on the equipment, but also reduces the comfort of passengers. There is a great risk that it will even capsize the ship in serious cases. So the stable operation of a ship is critical, and the stabilization has become the key study area. Many anti-rolling theories have been put forword and a variety of anti-rolling devices have been designed. The anti-rolling tank is an important device. It has the advantages of simple structure, low cost and reliable operation. When a ship sails at a slow speed, the performance of antirolling is particularly prominent, so it is widely used[1].

In order to verify whether the performance of anti-rolling tank meets the requirements or not, experiments are needed to be carried out. The anti-rolling tank swing bench is an experiment device to verify the requirements. It can simulate the motion of ship in waves. And it is a typical hydraulic position servo system, using the valve controlled hydraulic control method. An appropriate controller using the control strategy is designed to achieve a good position tracking performance. According to the ship parameters, the parameters of swing bench are adjusted by using similarity criterion. The swing bench installed with an appropriate anti-rolling tank can be used for testing. The performance of anti-rolling tank can be analyzed according to the experimental data.

The load of swing bench is the anti-rolling tank. The flow of water in tank is nonlinear, and there is clearance error in the transmission process. Due to the factors of mechanical precision, coupling of rolling motion and swaying motion, coupling of swing bench and 
tank, it is difficult to establish an accurate mathematical model of the system. When the load is fixed, the classic PID control effect is ideal. However, when the load changes, it is difficult to meet the requirements in a real system[2].

In previous studies, some controllers are designed to improve the accuracy and robustness of the hydraulic position servo system. In reference[3], a PID controller is designed, and the method is easy to be realized, but the control effect is not good when the load changes. In reference[4], in order to compensate interference torque owing to the fluid sloshing in tank, composite controller is designed based on PID disturbance observer and speed feedforward. It expands system bandwidth, but there are some phase lag between the input and output. So it can not meet the experimental requirements of smaller scale anti-rolling tank model. In reference[5], it designs a robust controller to achieve good effect by converting the deviation to the uncertainty of the model. However, it is simulated on a computer and not verified in actual system. In reference[6], a controller based on robust loop shaping is designed to achieve a good control performance. But the control parameters are fixed and are not self-adjusted against the change of load parameters.

In order to solve the problems of the swing bench, the frequency characteristic of system is needed to be analyzed. At the same time, the controller parameters are tuned according to the performance index of the actual situation. Through the observation of tracking ability, the parameters are adjusted further. This process is very cumbersome. The error is large and it is not easy to realize. A great deal of practical experience is needed. This paper introduces the BP neural network PID control strategy, and PID controller parameters are constant adjusted using learning ability of neural network. The self-tuning of parameters is realized to achieve the desired purpose[7].

To design the controller, model is needed to be established, simulated and tested. The whole process is tedious and prone to error. The virtual instrument LabVIEW has data acquisition and processing modules, and it also can complete the simulation work. The controller is designed in LabVIEW to achieve closed-loop control and data acquisition. When the system parameters change, the controller parameters are adjusted through the simulation program. After it is simulated to achieve the desired objectives, the controller parameters are loaded into the actual system. The mathematical model of hydraulic position servo system is of universal applicability. The model can be estabilshed by changing a few parameters. So the study of this paper can provide a practical method for other similar systems.

\section{Mathematical models for control}

The rolling hydraulic servo system of the swing bench comprises controller, servo valve, swing cylinder, test platform and angular sensor[8], concrete structure is shown in Figure 1.

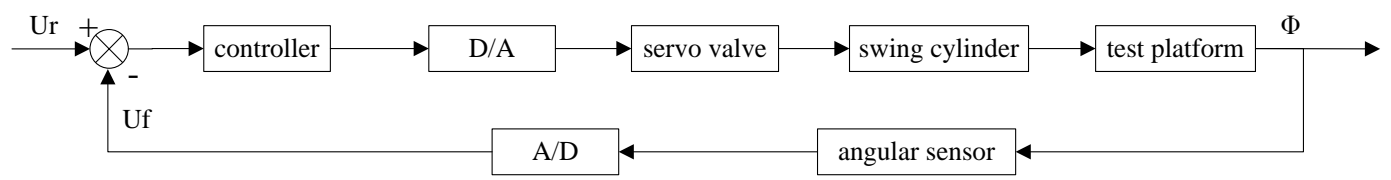

Figure 1. Rolling Hydraulic Servo System

(1) Flux equation of hydraulic valve

$$
Q_{\mathrm{L}}=K_{\mathrm{q}} X_{\mathrm{v}}-K_{\mathrm{c}} P_{\mathrm{L}}
$$


Where: $K_{q}$ is the flux gain of slide valve at steady state working point. $X_{v}$ is the displacement of slide valve's spool. $K_{c}$ is the flux-valve coefficient. $P_{\mathrm{L}}$ is the both chambers difference of hydraulic cylinder, namely the drop of load.

(2) Continuity equation of hydraulic cylinder

$$
Q_{\mathrm{L}}=A \frac{d y}{d t}+C_{\mathrm{tc}} p_{\mathrm{L}}+\frac{V_{\mathrm{t}}}{4 \beta_{\mathrm{e}}} \cdot \frac{d p_{\mathrm{L}}}{d t}
$$

Where: $A$ is the effective area of plunger in the hydraulic cylinder. $C_{\mathrm{tc}}$ is the total leakage coefficient of hydraulic cylinder. $V_{\mathrm{t}}$ is the whole volume of hydraulic cylinder. $\beta_{e}$ is the effective elastic modulus of volume.

(3) Hydraulic cylinder and load force balance equation

$$
A p_{\mathrm{L}}=m \frac{d^{2} y}{d t^{2}}+B_{c} \frac{d y}{d t}+K y+F_{L}
$$

Where: $m$ is the total mass of plunger and load. $B_{c}$ is the viscous damping coefficient of plunger and load. $F_{L}$ is the random load force on the plunger. $K$ is the load spring rate.

The equation above can be translated by Laplace transformation as follow:

$$
Y=\frac{\frac{K_{q}}{A} X_{v}-\frac{K_{c e}}{A^{2}}\left(\frac{V_{t}}{4 \beta_{e} K_{c e}} s+1\right) F}{\frac{m V_{t}}{4 \beta_{e} A^{2}} s^{3}+\left(\frac{m K_{c e}}{A^{2}}+\frac{B_{c} V_{t}}{4 \beta_{e} A^{2}}\right) s^{2}+\left(\frac{K V_{t}}{4 \beta_{e} A^{2}}+\frac{B_{c} K_{c e}}{A^{2}}+1\right) s+\frac{K K_{c e}}{A^{2}}}
$$

Where: $K_{c e}=K_{c}+C_{t c}$ is the whole flow-pressure coefficient.

As the load of test bench is inertia load, and elastic load is very small which could be ignored, then it can be regarded as $K_{c}=0$ and then $K_{c e}=C_{t c} . B_{c}$ is very small which could be ignored.

The equation (4) is simplified as:

$$
Y=\frac{\frac{K_{q}}{A} X_{v}-\frac{K_{c e}}{A^{2}}\left(\frac{V_{t}}{4 \beta_{e} K_{c e}} s+1\right) F}{s\left(\frac{s^{2}}{\omega_{h}^{2}}+\frac{2 \zeta_{h}}{\omega_{h}} s+1\right)}
$$

Where: $\omega_{h}$ is the natural frequency of hydraulic pressure. $\zeta_{h}$ is the hydraulic damping ratio.

$$
\begin{gathered}
\zeta_{h}=\frac{K_{c e}}{A} \sqrt{\frac{\beta_{e} m}{V_{t}}} \\
\omega_{h}=\sqrt{\frac{4 \beta_{e} A^{2}}{V_{t} m}}
\end{gathered}
$$

(4) The transfer function of servo valve

Because the system requires normal power, the servo valve controlling method is used. The bandwidth of servo valve is over 10 times greater than execution partial natural 
frequency of part hydraulic. The dynamic nature of servo valve is regarded as proportional component $K_{Q}$ :

$$
K_{Q}=\frac{Q(s)}{I(s)}
$$

Where: $Q(s)$ is the output of the servo valve. $I(s)$ is the input of the servo valve.

(5) Transfer function of servo amplifier and sensor

The servo amplifier bandwidth of forward path is much wider than the natural frequency of hydraulic pressure, so the servo amplifier of forward path is somehow considered as the proportional component $K_{a}$. The angular sensor and displacement sensor are test element device of double-freedom position system. Similarly, it could be regarded as the proportional component, noted as $K_{f}$ and $K_{d}$ respectively.

The open-loop transfer function of rolling system:

$$
P_{r}=K_{a} K_{f} \frac{K_{Q}}{A} \frac{1}{k} \frac{1}{s\left(\frac{s^{2}}{\omega_{h}^{2}}+\frac{2 \zeta_{h}}{\omega_{h}} s+1\right)}
$$

Where: $k$ is swinging cylinder's transmission radius.

\section{Controller Design of BP Neural Network PID}

\subsection{Control Analyse}

Over years, the ideal effect of classic PID control has been widely validated by many systems. The key of designing classic PID controller is to find the best combined PID parameters and to make the system achieve the desired control performance. Usually it can get the appropriate parameters after a series of complicated computing. However, when the appropriate parameters in theory are applied to actual system, it can not achieve the expected control effect. The reasons are due to the influence of the error and nonlinear of mathematical model, the mechanical precision of actual system and structure parameters changing and other factors. Therefore, in practical application, the parameters are usually adjusted by trial method. The process is inconvenient and less operation. So it is necessary to select the appropriate control strategy to improve the design process.

The BP neural network control strategy has the ability of self-learning. It can constantly change its structure parameters according to inputs and outputs, which makes the system achieve the expected control effect. Therefore, it is considered to combine the classic PID control and BP neural network control. The output of the neural network is provided for PID controller parameters. It can automatically acquire PID control parameters by using the learning ability of neural network.

This control method is very suitable for the hydraulic servo system of swing bench. First of all, this control method does not need troublesomely calculate to obtain the parameters. And it does not rely too much on the precise mathematical model. Secondly, it is less relevant to time-varying, nonlinearity and the parameters change and other factors. Because the controller parameters are changed following the change of system parameters, the dynamic controller is suitable to solve the problems of swing bench.

\subsection{Structure of Controller}

BP neural network PID control is combined of BP neural network and classic PID control, its structure is shown in Figure 2. 


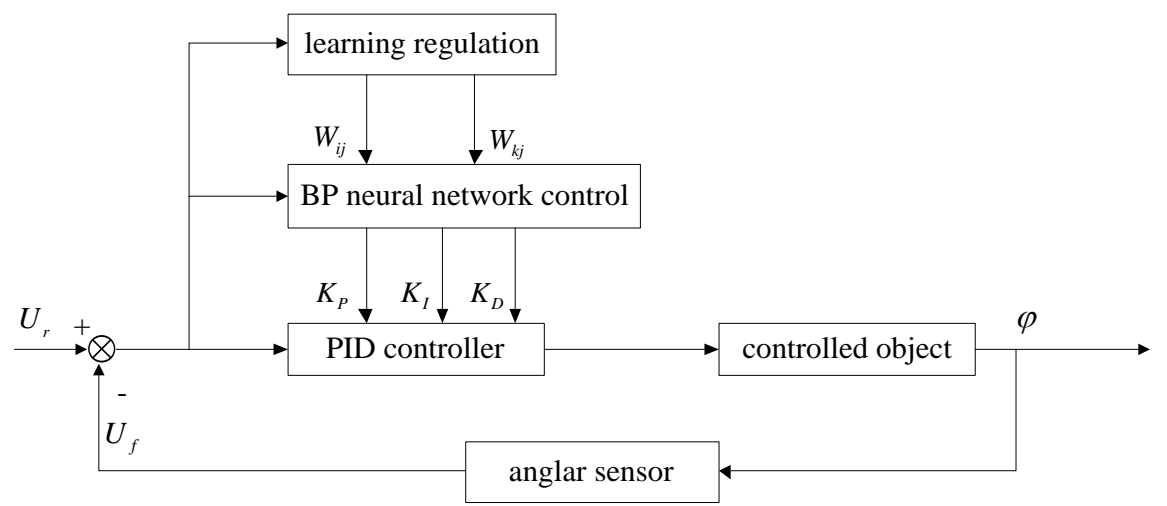

Figure 2. Structure of BP Neural Network PID Controller

The design of BP neural network needs to adjust the connection weights online according to the input and output of swing bench. Therefore, choose $x=[e(k), 1]^{T}$ as the input signal. So the BP neural network is the structure of $2 \times 5 \times 3$. Namely there are 2 neurons in input layer, 5 neurons in hidden layer and 3 neurons in output layer.

\subsection{Learning Algorithm}

The control objective of swing bench is to make the output of the rolling angle to track the given control signal [9]. So output error of energy could be as the objective function in the learning algorithm of neural network:

$$
E(k)=\frac{1}{2}(r(k)-y(k))^{2}
$$

The weight adjustment of neural network is to make the target function to minimum. Network connection weights are corrected in accordance with the steepest gradient descent method. Learning rate is $\eta$ and learning rules are as follows:

(1) The weights adjustment of hidden layer to the output layer

$$
\Delta w_{l i}^{(3)}(k)=-\eta \frac{\partial E(k)}{\partial w_{l i}^{(3)}}+\alpha \Delta w_{l i}^{(3)}(k-1)
$$

Where: $\alpha \Delta w_{l i}^{(3)}(k-1)$ is inertia. $\alpha$ is inertia coefficient.

It is a drawback of BP neural network, whose convergence speed is slow. In real time systems, quick response is pursued. The slow convergence speed would cause low speed of PID parameters adjusting, and the controller can't cope with changes and disturbances of swing bench. The introduction of inertia can increase the speed of convergence effect.

$$
\frac{\partial E(k)}{\partial w_{l i}^{(3)}}=\frac{\partial E(k)}{\partial y(k)} \frac{\partial y(k)}{\partial \Delta u(k)} \frac{\partial \Delta u(k)}{\partial o_{l}^{(3)}} \frac{\partial o_{l}^{(3)}}{\partial n e t_{l}^{(3)}} \frac{\partial n e t_{l}^{(3)}}{\partial w_{l i}^{(3)}}
$$

Where: $\Delta u(k)$ is the output of the PID controller.

Then, it is obtained:

$$
\begin{aligned}
& \frac{\partial \Delta u(k)}{\partial o_{1}^{(3)}}=\frac{\partial \Delta u(k)}{\partial K_{P}}=e(k)-e(k-1) \\
& \frac{\partial \Delta u(k)}{\partial o_{2}^{(3)}}=\frac{\partial \Delta u(k)}{\partial K_{I}}=e(k) \\
& \frac{\partial \Delta u(k)}{\partial o_{3}^{(3)}}=\frac{\partial \Delta u(k)}{\partial K_{D}}=e(k)-2 e(k-1)+e(k-2)
\end{aligned}
$$


In the design of BP neural network PID controller process, the value of $\frac{\partial y(k)}{\partial \Delta u(k)}$ is not sure. So it is replaced by the sign function $\operatorname{sign}\left(\frac{\partial y(k)}{\partial \Delta u(k)}\right)$. Because $\frac{\partial y(k)}{\partial \Delta u(k)}$ and $\operatorname{sign}\left(\frac{\partial y(k)}{\partial \Delta u(k)}\right)$ has the same symbol, the replacement will not change the weight adjustment direction, and it would only change the speed of adjustment. The end result is same after several adjustments. The adjustment speed can be compensated by changing the value of $\eta$ appropriately.

After simplification, the weights adjustment rules of hidden layer and output layer can be obtained:

$$
\begin{aligned}
& \Delta w_{l i}^{(3)}(k)=\eta \delta_{l}^{(3)} o_{i}(k)+\alpha \Delta w_{l i}^{(3)}(k-1) \\
& \delta_{l}^{(3)}=e(k) \operatorname{sign}\left(\frac{\partial y(k)}{\partial \Delta u(k)}\right) \frac{\partial \Delta u(k)}{\partial o_{l}^{(3)}(k)} f^{\prime} \quad\left(n e t_{l}^{(3)}(k)\right) \quad(l=1,2,3) \\
& w_{l i}^{(3)}(k)=\Delta w_{l i}^{(3)}(k)+w_{l i}^{(3)}(k-1)
\end{aligned}
$$

(2) The weights adjustment of input layer to the hidden layer

The weights adjustment rules are derived as above, and it is available:

$$
\begin{aligned}
& \Delta w_{i j}^{(2)}(k)=\eta \delta_{i}^{(2)} x_{j}(k)+\alpha \Delta w_{i j}^{(2)}(k-1) \\
& \delta_{i}^{(2)}=g^{\prime}\left(\text { net }_{i}^{(2)}(k)\right) \sum_{l=1}^{3} \delta_{i}^{(3)} w_{i j}^{(3)}(k) \quad(i=1,2, \ldots, 5) \\
& w_{i j}^{(2)}(k)=\Delta w_{i j}^{(2)}(k)+w_{i j}^{(2)}(k-1)
\end{aligned}
$$

\subsection{Design Steps of Controller}

Through the above analysis, the design steps of BP neural network PID controller are as follows:

(1) Initialize. After the structure of the BP neural network is selected, all of connection weights should be given the initial value. Then the values of learning rate $\eta$ and inertia coefficients $\alpha$ should be set up in the algorithm. The system uses the network structure of $2 \times 5 \times 3, \eta=0.001, \alpha=0.01$. Each initial values of connection weight is a random number.

(2) Obtain the input signal of BP neural network and calculate the output. The input and output of system are obtained through the acquisition module and the error is calculated as the input signal. Then output of network is calculated according to the feedforward method.

(3) Calculate the output of controller. After controller parameters and input are obtained, controller output is calculated in accordance with the incremental PID control and the control signal of the controlled object is obtained.

(4) Adjust the weighted coefficient. The connection weights of the network are adjusted.

(5) Repeat steps 2 4. 


\subsection{Controller Implement in LabVIEW}

In order to realize controller design rapidly and effectively, appropriate virtual instrument is needed to make it suitable for hydraulic position system of swing bench. LabVIEW can be convenient for modeling, simulation, data acquisition and processing. Therefore, it can be competent for the work.

BP neural network controller is realized by calling MATLAB program in LabVIEW. The control is divided into three parts. The first part is to calculate the parameters of the controller. The second part is to calculate the output of PID controller. The third part is to adjust the neural network weights. There are flow diagram of real-time control program as shown in Figure 3.

We can choose to enter the control system or data processing system by the list of LabVIEW panel. Similarly, the input signal type and the specific parameters can also be adjusted by the way.

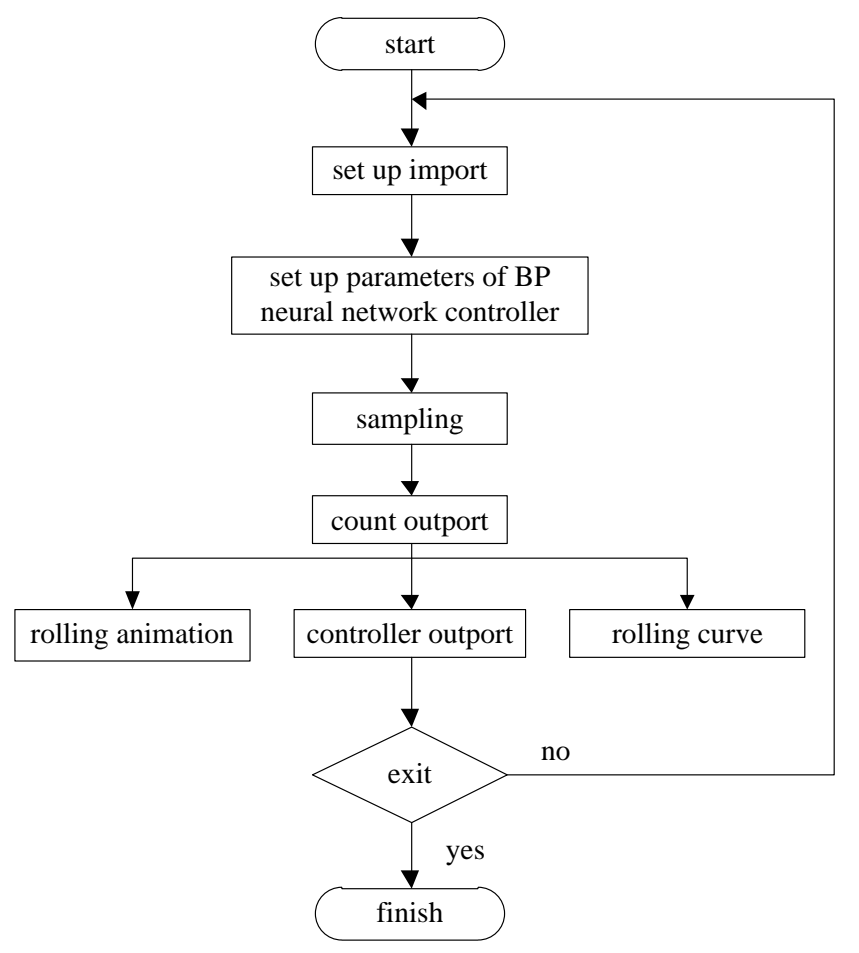

Figure 3. Flow Diagram of Real-Time Control Program

The LabVIEW control panel of system is shown in Figure 4.

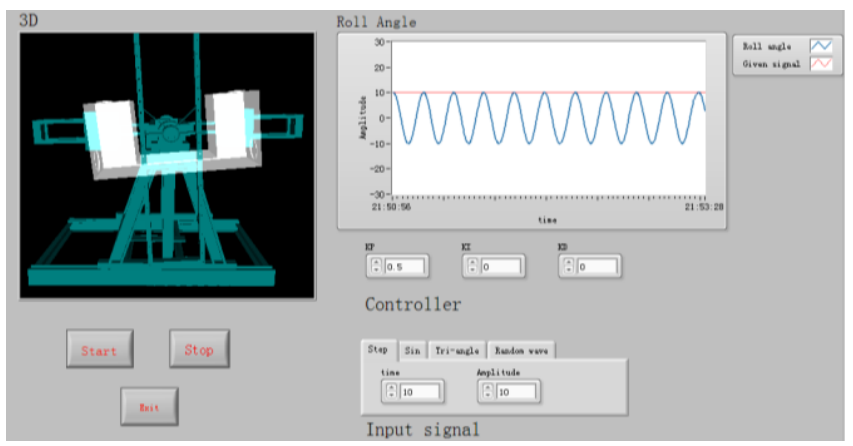

Figure 4. LabVIEW Control Panel of System 


\section{Experiment}

\subsection{Experimental Object}

(1) Swing bench. To avoid the influence of the scale effect, the swing bench is designed by a certain scale according to resilience and disturbing force of ship and the anti-rolling tank. The maximum achievable rolling range is $30^{\circ}$. As is shown in Figure 5, and the main parameters of system is shown in Table 1.

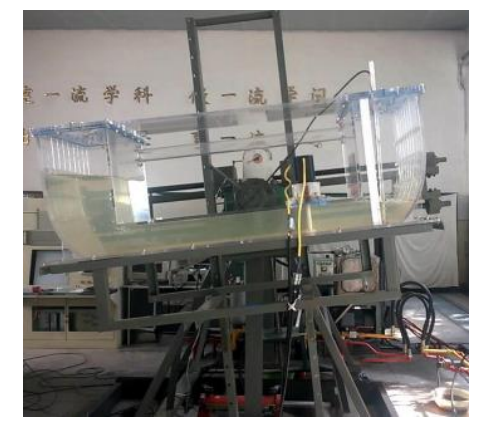

Table 1. Parameters of System

\section{Figure 5. Anti-rolling Tank Swing Bench}

\begin{tabular}{lll}
\hline Coefficient & Unit & Value \\
\hline$K_{f}$ & $/$ & 55 \\
$K_{Q}$ & $/$ & 0.145 \\
$k$ & $\mathrm{~m}$ & 0.0549 \\
$\omega_{h}$ & $\mathrm{rad} / \mathrm{s}$ & 47.51 \\
$\zeta_{h}$ & $/$ & 0.2 \\
$A$ & $\mathrm{~m}^{2}$ & $6.234 * 10^{-3}$ \\
\hline
\end{tabular}

(2) Anti-rolling tank. In this paper, the width of ship's actual equipment tank is $7.2 \mathrm{~m}$. The maximum width of tank model is $0.8 \mathrm{~m}$ for the swing bench. If the tank model scale is too small, it would cause the larger scale effect and great experimental error. The reasonable scale ratio is $10: 1$ and then the choice of width is $0.72 \mathrm{~m}$.

\subsection{Experimental Procedure}

Swing bench is a position servo system, which needs achieve fast and accurate tracking of the dynamic signal and has good robustness. Testing methods mainly include the forced oscillation test and random excitation test. Namely it is verified by regular waves and irregular waves. To test the real time control effect, tracking experiments are carried out using the step signal, sinusoidal signal and random roll angle signal.

During the experiment, it can be realized the input of the signals, the tuning of the controller, the 3D real time display and data processing output can be set by the control panel. The learning rate and the inertia coefficient etc. can also be set.

\subsection{Result Analysis}

During the experiment, the system becomes unstable after adding the integral link. Because the integral link makes the system increase of $90^{\circ}$ phase lagged, it substantially reduces phase margin. When the integral time constant is very little, the system is stable. In addition, the integral link is to improve the integral type of system and to reduce the steady-state error. The original system is a I type system, so the effect is not obvious after adding integral link. Therefor, the controller chooses PD structure.

The step signal, sinusoidal signal and random roll angle signal response curve and controller parameters curve, are shown in Figure 6. (a), (c), and (e) are the three response curves. And (b), (d), and (f) are the corresponding controller parameters curves. 


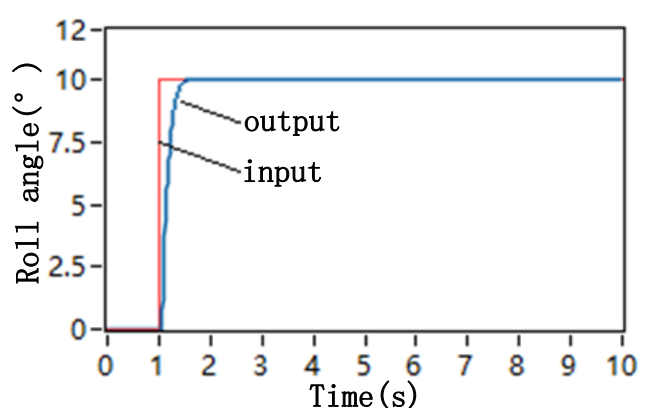

(a)

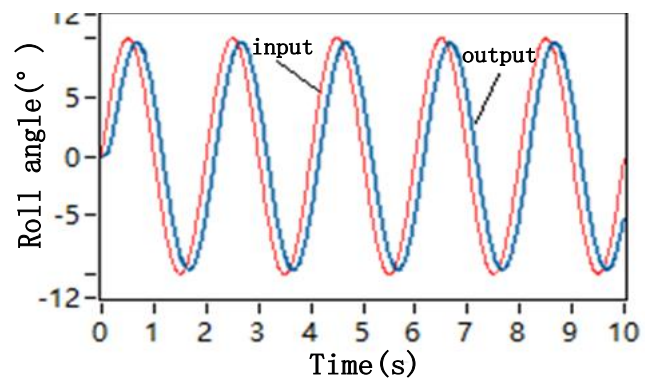

(c)

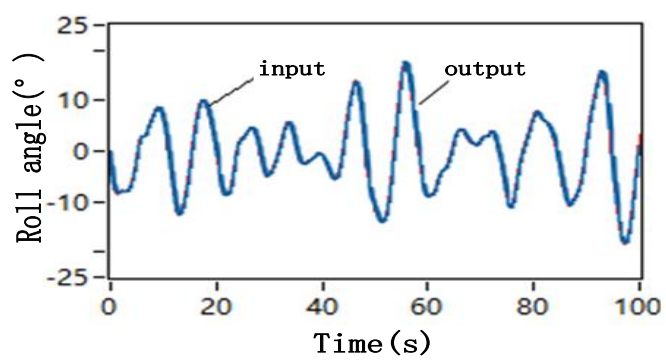

(e)

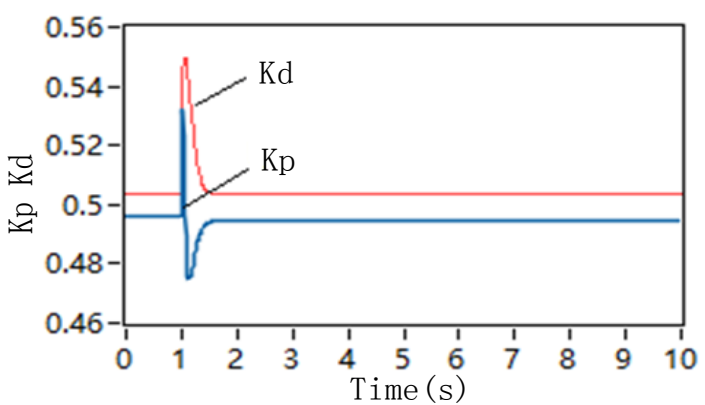

(b)

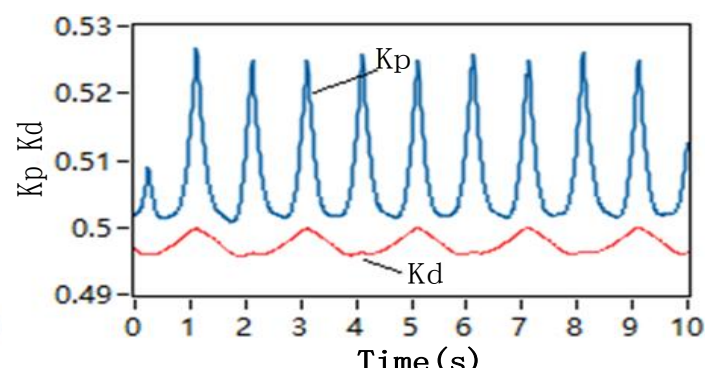

(d)

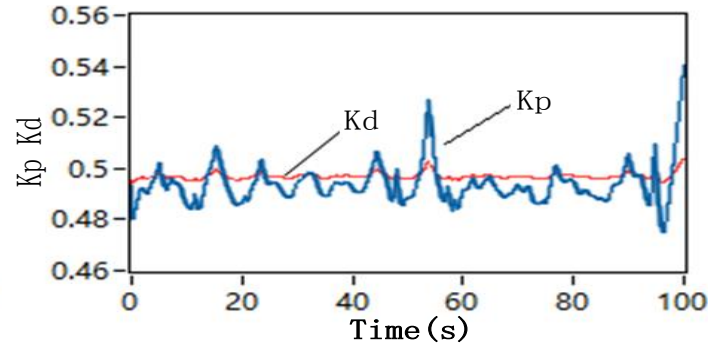

(f)

Figure 6. Response Curve and Controller Parameters Curve

(1) Step response Experiment. It can be obtained from Figure 6 (a) and (b): the system output is no overshoot, fast and stable in $0.5 \mathrm{~s}$ by using the designed controller. The controller parameters vary all the time. When the deviation is larger, the parameters of proportion link and differential link are relatively larger and the system is provided with a good fast performance, which can quickly reduce the deviation. When the deviation is smaller, the parameters become small, which make the system more stable and reduce or even eliminate the overshoot. Finally, when the stability of deviation is zero, the controller parameters converge to fixed values.

(2) Sinusoidal Response Experiment. It can be obtained from Figure 6 (c) and (d): the tracking performance of system is also quite good for sinusoidal signal which varies slowly and the parameters are continually changing with the change of deviation. Periodic tracking response has been well verified.

(3) Random Roll Angle Response Experiment. When the significant wave height of simulation of ocean waves is $5 \mathrm{~m}$, angle of $90^{\circ}$ and the ship speed is $10 \mathrm{kn}$, the roll angle signal is generated [10].

Because the ship is usually sailing in the long crested waves. The long crested waves are adopted to simulate the irregular waves. So it is closer to the actual ocean situation to test the dynamic performance of swing bench using the random roll angle signal. 
It can be concluded from Figure 6 (e) and (f): the system can quickly and accurately track for the simulation in real sea off random roll angle signal. The controller parameters are continuously adjusted in the dynamic tracking of input signal. It can meet the requirements of actual system.

\section{Conclusion}

Aimed at the uncertainty problems of swing bench, this paper designs a controller using BP neural network PID method based on LabVIEW to improve the servo system's adaptability and possess the performance of approaching any continuous bounded nonlinear function. The controller parameters are adjusted by BP neural network automatically, and the favourable stability and tracking accuracy are able to be provided.

LabVIEW makes it flexible and comprehensive in control and data processing of the system. By using the control panel, the operation is simpler and the experiment is more effective. The user interface is friendly and easy to be expanded. It can provide a good reference for controller design of hydraulic servo system of other types.

\section{Acknowledgments}

The authors acknowledge the contribution of the National Natural Science Foundation of China (61304076), the Technological Innovation Foundation of Harbin (RC2013QN009035), and the Experimental Education Reformation and Technological Research of Harbin Engineering University (SYJG20130411).

\section{References}

[1] Songtao Zhang, Jian Lu, Lihua Liang and Mingxiao Sun. The Control Method and Test of Anti-Rolling Tank Based on Half Period Prediction. Techniques of Automation and Applications. 33(4): 6-9, (2014)

[2] Liang Lihua, Zhang Songtao, He Changjiu. Predictive control and stabilizing effect analysis of controlled passive tank. The 9th IEEE International Conference on Mechatronics and Automation, (2012) 2547-2552, Chengdu

[3] Liang Lihua, Yang Peng, Ma Jie, Li Guobin. Application of fuzzy-PID controller to the rolling position system of ship testing platform. Ship Engineering, 1:32-35, (2006)

[4] ZHANG Song-tao, LI Xin-ning, LIANG Li-hua, WANG Na. Position Servo System Compound Control Based on Disturbance Observer. Chinese Hydraulics \& Pneumatics. (11): 44-47, (2013)

[5] Shi Hongyu, Liang Lihua, Zhang Songtao. Application of inverse system on ship motion simulation bench. 2010 IEEE International Conference on Information and Automation, (2010) 1376-1381, Harbin

[6] Zhang S, Sun M, Liang L and Li X. Loop shaping-based robust controlin hydraulic servo system. Mechatronic Sciences, 2013 IEEE International Conference on Electric Engineering and Computer, (2013) 116-119, Shenyang

[7] Zhang Songtao, Liang Lihua, Sheng Xiaoliang. Parameters optimization of controlled anti-rolling tank based on multi-objective optimal method. 2012 IEEE International Conference on Automation and Logistics, (2012) 201-206, Zhengzhou

[8] Yu Lijun, Meng Xiangzhen. Design and simulation of fuzzy immune controller of fin stabilizer-passive anti-rolling tank integrated stabilization system. 2nd International Conference on Information Science and Engineering, (2010) 5047-5050, Beijing

[9] Holden, Christian, Tristan Perez, and Thor I. Fossen. A Lagrangian approach to nonlinear modeling of anti-roll tanks. Ocean Engineering 38.2: 341-359, (2011)

[10] Liang Lihua, Zhang Songtao, Shi Hongyu. Fluid Simulation of Anti - Rolling Tank Based on CFD. Computer Simulation, 12(29):401-405, (2012) 